\title{
Trait anhedonia is associated with reduced reactivity and connectivity of mesolimbic and paralimbic reward pathways
}

\author{
Jennifer Keller ${ }^{\mathrm{a}, 1}$, Christina B. Young ${ }^{\mathrm{b}, 1}$, Elizabeth Kelley ${ }^{\mathrm{a}}$, Katherine Prater $^{\mathrm{c}}$, \\ Daniel J. Levitin ${ }^{\mathrm{d}}$, Vinod Menon ${ }^{\mathrm{a}, \mathrm{e}, \mathrm{f}, *}$ \\ a Department of Psychiatry \& Behavioral Sciences, Stanford University School of Medicine, Stanford, CA 94305, USA \\ ${ }^{\mathrm{b}}$ Department of Psychology, Northwestern University, Evanston, IL 60208, USA \\ ${ }^{\mathrm{c}}$ Neuroscience Program, University of Michigan, Ann Arbor, MI 48109, USA \\ ${ }^{\mathrm{d}}$ Department of Psychology, McGill University, Montreal, QC H3A 1B1 Canada \\ e Program in Neuroscience, Stanford University School of Medicine, Stanford, CA 94305, USA \\ ${ }^{\mathrm{f}}$ Department of Neurology \& Neurological Sciences, Stanford University School of Medicine, Stanford, CA 94305, USA
}

\section{A R T I C L E I N F O}

\section{Article history:}

Received 17 October 2012

Received in revised form

15 May 2013

Accepted 16 May 2013

\section{Keywords:}

Anhedonia

Mesolimbic reward system

Nucleus accumbens

Insula

Orbitofrontal cortex

Ventral tegmental area

\begin{abstract}
A B S T R A C T
Anhedonia is the inability to experience pleasure from normally pleasant stimuli. Although anhedonia is a prominent feature of many psychiatric disorders, trait anhedonia is also observed dimensionally in healthy individuals. Currently, the neurobiological basis of anhedonia is poorly understood because it has been mainly investigated in patients with psychiatric disorders. Thus, previous studies have not been able to adequately disentangle the neural correlates of anhedonia from other clinical symptoms. In this study, trait anhedonia was assessed in well-characterized healthy participants with no history of Axis I psychiatric illness. Functional magnetic resonance imaging with musical stimuli was used to examine brain responses and effective connectivity in relation to individual differences in anhedonia. We found that trait anhedonia was negatively correlated with pleasantness ratings of music stimuli and with activation of key brain structures involved in reward processing, including nucleus accumbens (NAc), basal forebrain and hypothalamus which are linked by the medial forebrain bundle to the ventral tegmental area (VTA). Brain regions important for processing salient emotional stimuli, including anterior insula and orbitofrontal cortex were also negatively correlated with trait anhedonia. Furthermore, effective connectivity between NAc, VTA and paralimbic areas, that regulate emotional reactivity to hedonic stimuli, was negatively correlated with trait anhedonia. Our results indicate that trait anhedonia is associated with reduced reactivity and connectivity of mesolimbic and related limbic and paralimbic systems involved in reward processing. Critically, this association can be detected even in individuals without psychiatric illness. Our findings have important implications both for understanding the neurobiological basis of anhedonia and for the treatment of anhedonia in psychiatric disorders.
\end{abstract}

(C) 2013 Elsevier Ltd. All rights reserved.
Anhedonia, or the inability to experience pleasure from normally pleasant stimuli and life events, is a common symptom of many psychiatric disorders, including major depressive disorder and schizophrenia (Chapman et al., 1976). As a clinical symptom, anhedonia has a significant impact on the functioning of psychiatric patients and has been associated with psychosocial and functional disability in both depression and schizophrenia (Buckner et al.,

\footnotetext{
* Corresponding author. Department of Psychiatry \& Behavioral Sciences, Program in Neuroscience, Stanford University School of Medicine, Stanford, CA 94305, USA. Tel.: +1 650498 6737; fax: +1 6507367200 .

E-mail address: menon@stanford.edu (V. Menon).

1 These authors contributed equally to the study.
}

2008; Wolf, 2006). In psychiatric illnesses, anhedonia is not adequately treated by pharmacological and psychosocial interventions (Buckner et al., 2008; Horan et al., 2006; Wolf, 2006), and it persists even after the remission of other symptoms (Barkham et al., 1996; Kopta et al., 1994; Shelton and Tomarken, 2001). Although anhedonia has been studied more widely in clinical populations, individual differences in anhedonia are also present in non-clinical populations (Franken et al., 2007; Harvey et al., 2007). Little is currently known about the neurobiological basis of anhedonia, and addressing its dimensional aspects remains an important open question for both basic and clinical affective neuroscience.

The primary goal of our study was to investigate the neural correlates of trait anhedonia in healthy adults without a history of psychiatric illness. Both the recently conceptualized "liking" and 
"wanting" components of anhedonia (Berridge and Kringelbach, 2008; Horan et al., 2006) involve the mesolimbic reward system (MRS) and its associated pathways (Der-Avakian and Markou, 2012). The structural core of the MRS consists of the nucleus accumbens (NAc) and the ventral tegmental area/substantia nigra (VTA/SN) (Haber and Knutson, 2010; Knutson et al., 2001), which are connected by the medial forebrain bundle (MFB). The NAc, in particular, has been consistently linked to processing pleasurable stimuli, including those that do not involve explicit reward (PadoaSchioppa and Cai, 2011). The VTA is the site of mesolimbic dopamine neuron cell bodies that project to the NAc, and dopamine release by the VTA is known to be crucial for reward processing (Padoa-Schioppa and Cai, 2011). The MFB not only connects the VTA to the NAc, but also has distal connections to basal forebrain and frontal lobe regions that are important for reward and motivation (Coenen et al., 2012). Together, brain structures associated with the MRS and MFB are critical for reward seeking as well as maintaining equilibrium between positive and negative affective states (Coenen et al., 2011). Indeed, clinical studies using deep-brain stimulation on brain regions linked by the MFB have been shown to be effective in alleviating depression symptoms by increasing reward seeking desires (Coenen et al., 2011; Schoene-Bake et al., 2010). Brain imaging studies in humans have also reported a consistent pattern of MRS co-activation in response to various rewards, including food (Small et al., 2001), money (Knutson et al., 2001), humorous cartoons (Mobbs et al., 2003), music (Koelsch et al., 2006; Menon and Levitin, 2005), and pleasant pictures (Lane et al., 1997). Mesolimbic and mesocortical pathways involving the VTA, NAc, amygdala, OFC and medial prefrontal cortex are thought to be critical for reward processing, anticipation and learning (Buckholtz et al., 2010; Chiavaras et al., 2001; Schlaepfer et al., 2008), but little is known about the relation between anhedonia and functional interactions within mesolimbic and mesocortical pathways that link these regions.

The strong overlap between anhedonia and other clinical symptoms in disorders such as depression, has made it difficult to isolate the neural correlates of anhedonia. In other words, because anhedonia is often assessed within the context of psychiatric or neurological disorders, it is difficult to separate the effects of anhedonia from the effects of the disorder. In depressed patients, Dunn and colleagues (2002) reported negative correlations between anhedonia severity and response in subcortical regions, including the ventral striatum. Keedwell and colleagues (2005) also examined depressed patients and found significant positive and negative correlations between anhedonia severity and response to happy stimuli primarily within the ventromedial prefrontal cortex and striatum, respectively. They suggested that anhedonic depressed patients attend more closely to the rewarding stimulus in an attempt to enter a happy mood. However, depression severity scores were also correlated with neural activity of the ventral striatal structures. Thus, previous brain imaging studies have not been able to adequately disentangle anhedonia from other clinical symptoms.

Only one study to date has examined the association between trait anhedonia and brain activity in healthy controls. Harvey et al. (2007) used structural and functional brain imaging to examine hedonic capacity in non-clinical participants. They found that when participants viewed positively valenced pictures during an emotional memory task, trait anhedonia was positively correlated with activity in several structures, including the ventromedial prefrontal cortex, superior temporal gyrus, insula, superior parietal lobule and occipital cortex. Surprisingly, MRS and ventral striatal responses were not significantly correlated with trait anhedonia. More recently, Wacker and colleagues (2009) examined individual differences in hedonic capacity in a mixed sample of healthy adults and adults with psychiatric illness and found that anhedonia, but not depression or anxiety, correlated with reduced NAc activity in response to reward. The contradictory findings reported in these two studies may partly be attributable to methodological and sample differences. Harvey et al. (2007) used the Chapman physical anhedonia scale (Chapman et al., 1976) to determine hedonic capacity. On the other hand, Wacker and colleagues (2009) used the Mood and Anxiety Symptom Questionnaire anhedonic depression scale (MASQ-AD) (Watson et al., 1995a, 1995b) to measure anhedonia, which is a more recently developed and encompassing assessment of anhedonia, but anhedonia was not assessed in close temporal proximity to the brain imaging session. Furthermore, 25\% of their sample had a history of psychiatric illness, which highlights the potentially confounding effects of psychiatric disorders on anhedonia. Finally, neither study examined how functional interactions between the MRS and the rest of the brain are altered by trait anhedonia.

To overcome limitations of previous studies, we examined a well-characterized group of healthy adults without any history of psychiatric illness. Importantly, we used the positive affect factor of the MASQ-AD to assess trait anhedonia. Using factor analysis, the MASQ-AD has been shown to consist of an 8-item depression factor and a 14-item reverse scored positive affect factor (Nitschke et al., 2001; Watson et al., 1995a, 1995b). The items of the MASQ-AD positive affect factor inquire about wide-ranging situations (e.g., "Looked forward to things with enjoyment" and "Felt like I had a lot of interesting things to do") and thus encompass components beyond physical and social anhedonia. Thus, by using the positive affect factor of the MASQ-AD, we were able to more directly examine the neural basis of trait anhedonia. We also used music to effectively probe the MRS because it is a naturalistic "realworld" auditory stimulus that is relevant to everyday experiences that are impaired in individuals with trait anhedonia. Music represents a dynamic form of emotion and the conveying of emotion is considered to be the essence of music and the reason that most people report spending large amounts of time listening to music (Juslin and Sloboda, 2001). Music is capable of evoking exceptionally strong emotions (Koelsch et al., 2006), including feelings of pleasure (Krumhansl, 1997; Sloboda and Juslin, 2001) and frisson (Berridge and Kringelbach, 2008; Mesulam, 1998). Even passive music listening is known to evoke the same strong physiological changes, such as frisson, shivers, and heart rate changes that often accompany strong emotional stimuli (Panksepp, 1995).

Critically, because music elicits a robust response in the ventral striatum (Blood and Zatorre, 2001; Blood et al., 1999; Menon and Levitin, 2005), we hypothesized that music would help capture trait anhedonia effects that might be missed by other less emotionally evocative and pleasurable stimuli. Both PET and fMRI have shown that activity in brain regions thought to be involved in reward/motivation and emotion, including striatum, midbrain, amygdala, orbitofrontal cortex (OFC) and ventromedial prefrontal cortex, is related to the subjective experience of music listening (Blood and Zatorre, 2001; Blood et al., 1999; Mitterschiffthaler et al., 2007). Research using fMRI connectivity analysis have further shown that compared to spectrally matched scrambled music, listening to music strongly modulated activity in a network of MRS structures including the NAC and the VTA/SN as well as associated structures in the hypothalamus, insula and OFC, which are thought to be involved in regulating autonomic and physiological responses to rewarding and emotional stimuli (Menon and Levitin, 2005). More recently, Salimpoor et al. (2011) combined PET with fMRI to demonstrate dopamine release in multiple MRS structures in conjunction with fMRI activation during music listening. These results suggest that listening to pleasant music evokes robust and reliable response in the MRS as well as in related structures such as the ventromedial prefrontal cortex and insula. 
In the present study, we used fMRI to examine brain response and effective connectivity of the MRS in relation to trait anhedonia. We hypothesized that individual differences in anhedonia would be related to MRS reactivity. Specifically, we predicted that trait anhedonia would be inversely correlated with activity in the NAc, VTA and other MRS nuclei linked by the MFB (Coenen et al., 2009,2011 ) during the processing of positively valenced, pleasurable music stimuli. In addition, we used psychophysiological interaction analysis (PPI) to examine MRS effective connectivity independent of overall task-related activation. We predicted that effective connectivity of the MRS and paralimbic areas including the OFC and insula (Mesulam, 1998) that integrate and regulate reward processing (Berridge and Kringelbach, 2008) would also be negatively correlated with trait anhedonia. Taken together, these findings would provide novel evidence that crucial brain pathways associated with reward processing are negatively impacted by trait anhedonia even in individuals without psychiatric illness.

\section{Materials and methods}

\subsection{Participants}

Twenty-one healthy adults (9 males and 12 females, ages 1852) were recruited through online and print advertisements (Table 1). Participants had no current or past Axis I psychiatric disorders, nor were they subsyndromal for any diagnosis. All subjects were required to have a score less than 5 on the Hamilton Depression Rating Scale (HAM-D) (Hamilton, 1960). Other exclusion criteria included major medical illnesses, history of seizures, head trauma, neurological illness, pregnancy, current or recent smoking habits, and metal in the body that precluded MRI. Participants were paid $\$ 75$. After complete description of the study was provided, written informed consent was obtained from each participant. The protocol was approved by the Stanford University School of Medicine Human Subjects Committee.

\subsection{Procedure}

Eligibility screening procedures for all subjects included the Structured Clinical Interview for the DSM-IV (First et al., 1997) and the HAM-D (Hamilton, 1960). Eligible individuals were scheduled for the imaging session. On the day of scanning, participants completed music preference ratings, the HAM-D, the Chapman physical and social anhedonia scales (Chapman et al., 1976), and the Mood and Anxiety Symptom Questionnaire Short Form (MASQ)

Table 1

Subject characteristics with depression and anhedonia ratings.

\begin{tabular}{lc}
\hline$N=21$ (9 males, 12 females) & Mean $\pm S D$ \\
\hline Age & $34.95 \pm 10.42$ \\
Years of education & $16.38 \pm 2.64$ \\
HAM-D & $0.76 \pm 1.30$ \\
MASQ & \\
Anhedonic depression scale & $50.05 \pm 8.81$ \\
$\quad$ Positive affect factor & $40.86 \pm 9.16$ \\
$\quad$ Depression factor & $9.19 \pm 1.69$ \\
General distress depressive symptoms scale & $13.90 \pm 2.07$ \\
Anxious arousal scale & $18.14 \pm 1.80$ \\
General distress anxiety symptoms scale & $12.62 \pm 1.94$ \\
Chapman & \\
Physical anhedonia scale & $8.29 \pm 6.27$ \\
Social anhedonia scale & $10.19 \pm 6.45$ \\
Total anhedonia scale & $18.48 \pm 11.69$
\end{tabular}

HAM-D = Hamilton Depression Rating Scale; MASQ = Mood and Anxiety Symptom Questionnaire; $S D=$ Standard Deviation.
(Watson et al., 1995a, 1995b), which has four separate subscales: anhedonic depression, anxious arousal, general distress depressive symptoms, and general distress anxiety symptoms. The anhedonic depression subscale is further composed of an 8-item depression factor and a reverse-scored 14-item positive affect factor (Nitschke et al., 2001; Watson et al., 1995a, 1995b). Trait anhedonia was assessed using the positive affect factor of the MASQ-AD, which has scores ranging from 14 (indicating no anhedonia) to 70 (indicating significant levels of anhedonia) and shows convergent validity with the Chapman physical anhedonia scale.

\subsection{Stimuli}

Stimuli were chosen based on a previously published study (Menon and Levitin, 2005) and consisted of three musical pieces that were likely familiar and three that were likely unfamiliar (stimuli available at: http://www.scsnl.stanford.edu). Music stimuli and scrambled versions were each presented for $23 \mathrm{~s}$, followed by a $24 \mathrm{~s}$ rest epoch. The scrambled music controlled for low-level acoustic features and attention to complex auditory events (see Supplementary materials). In our previous study, an independent group of participants rated these classical music clips as pleasant and their scrambled music counterparts as relatively unpleasant (Menon and Levitin, 2005).

\subsection{Experiment}

Participants listened and responded with a button-press at the start and end of each music epoch. Active responses ensured that participants engaged and attended to the task. Auditory stimuli were presented binaurally using a custom-built magnet compatible system (see Supplementary materials) and the volume was individually set at a comfortable listening level prior to beginning the study.

\subsection{Post-scan questionnaire}

Following the fMRI session, participants listened to the pieces again and rated the original and scrambled music pieces on a 9point Likert scale $(-4$ to +4$)$ on ten different bipolar semantic differentials: exciting-calm, unpleasant-pleasant, tense-relaxed, annoying-unannoying, dissonant-consonant, angry-peaceful, happy-sad, moving-unmoving, boring-interesting, and unfamiliarfamiliar (Table 2).

Table 2

Mean music stimulus ratings.

\begin{tabular}{lrll}
\hline Scale & $\begin{array}{l}\text { Music } \\
\text { rating } \pm S D\end{array}$ & $\begin{array}{l}\text { Scrambled music } \\
\text { rating } \pm S D\end{array}$ & $\begin{array}{l}\text { Music vs. scrambled } \\
\text { music }^{\mathrm{a}}\end{array}$ \\
\hline $\begin{array}{c}\text { Unpleasant }- \\
\text { pleasant }\end{array}$ & $1.92 \pm 0.99$ & $-1.99 \pm 1.08$ & $t(17)=14.15, p<.001$ \\
$\begin{array}{c}\text { Annoying - } \\
\text { unannoying }\end{array}$ & $1.86 \pm 1.22$ & $-2.06 \pm 1.19$ & $t(17)=10.58, p<.001$ \\
$\begin{array}{c}\text { Excited - calm } \\
\text { Tense - relaxed }\end{array}$ & $-0.96 \pm 0.99$ & $-1.41 \pm 1.01$ & $t(17)=2.37, p=.03$ \\
$\begin{array}{c}\text { Dissonant - } \\
\quad \text { consonant }\end{array}$ & $1.35 \pm 1.02$ & $-2.09 \pm 1.04$ & $t(17)=5.84, p<.001$ \\
$\begin{array}{c}\text { Angry - peaceful } \\
\text { Happy - sad }\end{array}$ & $0.80 \pm 0.93$ & $-0.41 \pm 0.84$ & $t(15)=4.60, p<.001$ \\
Moving - & $-0.82 \pm 0.72$ & $-0.06 \pm 0.66$ & $t(17)=-2.71, p=.015$ \\
$\quad$ unmoving & & $0.80 \pm 1.52$ & $t(17)=-7.48, p<.001$ \\
Boring - & $2.08 \pm 0.84$ & $-0.28 \pm 1.29$ & $t(17)=7.91, p<.001$ \\
$\quad$ Interesting & & & \\
\hline
\end{tabular}

Ratings were conducted on a 9-point Likert scale $(-4$ to +4$)$. For all bipolar pairs listed above, the first construct has a value of -4 and the second has a value of +4 . For example, unpleasant-pleasant ratings, $-4=$ unpleasant and $+4=$ pleasant.

a Paired samples $t$-test. 


\section{6. fMRI data acquisition}

Functional images were acquired on a 3T GE Signa scanner using a standard GE whole-head coil (software Lx 8.3). Detailed scan parameters are provided in Supplementary materials.

\section{7. fMRI data analysis}

\subsubsection{Preprocessing}

The first two time points were not analyzed to allow for signal equilibration effects. A linear shim correction was applied separately for each slice during reconstruction using a magnetic field map acquired automatically by the pulse sequence at the beginning of the scan (Glover and Lai, 1998). Functional MRI data were then analyzed using SPM8 software (http://www.fil.ion.ucl.ac.uk/ $\mathrm{spm})$. Images were realigned to correct for motion, corrected for errors in slice-timing, spatially transformed to standard stereotaxic MNI space, resampled every $2 \mathrm{~mm}$ using sinc interpolation and smoothed with a $6 \mathrm{~mm}$ full-width half-maximum Gaussian kernel to decrease spatial noise prior to statistical analysis. Translational movement in millimeters $(x, y, z)$ and rotational motion in degrees (pitch, roll, yaw) was calculated based on SPM8 parameters for motion correction of the functional images in each subject. No participant had movement greater than $3 \mathrm{~mm}$ translation or 3 degrees of rotation; therefore, none were excluded from analyses.

\subsubsection{Statistical analysis}

Task-related brain activation was identified using a general linear model and the theory of Gaussian random fields as implemented in SPM8. Individual subject analyses were first performed by modeling task-related conditions as well as 6 movement parameters from the preprocessing procedure mentioned above. Brain activity related to the task conditions (Music, Scrambled Music) was modeled using boxcar functions convolved with a canonical hemodynamic response function and a temporal dispersion derivative to account for voxel-wise latency differences in hemodynamic response. Low-frequency drifts at each voxel were removed using a high-pass filter $(0.5$ cycles $/ \mathrm{min})$ and serial correlations were accounted for by modeling the fMRI time series as a first-degree autoregressive process. Voxel-wise $t$-statistics maps for each condition were generated for each participant using a general linear model, along with the respective contrast images. Grouplevel activation was determined using individual subject contrast images and a second-level analysis of variance.

To better isolate specific MRS regions of interest (ROIs) we first examined brain areas that showed greater activation to music contrasted with the passive rest baseline. In this case, significant clusters of activation were determined using a voxel-wise statistical height threshold of $p<.01$, with family-wise error (FWE) corrections for multiple spatial comparisons at the cluster level $p<.05$ $(k=106$ voxels). We also examined the relation between trait anhedonia and brain activation using contrast images generated by comparing Music and spectrally matched Scrambled Music conditions with the MASQ-AD positive affect factor as a covariate in a general linear model. The other MASQ subscales (anxious arousal, general distress anxiety symptoms, and general distress depressive symptoms) and the depression factor of the MASQ-AD had minimal variance, precluding examination of their relationship to brain activity. Both positive and negative correlations in brain activation with trait anhedonia were assessed. Voxel-wise $t$-statistics maps were generated and cortical clusters with significant correlations were determined using a voxel-wise statistical height threshold of $p<.01$, with FWE corrections for multiple spatial comparisons at the cluster level $p<.05$ ( $k=106$ voxels). Subcortical clusters were thresholded at a height threshold of $p<.05$ with FWE corrections at the cluster level of $p<.05$ ( $k=515$ voxels). A less stringent statistical threshold was used for subcortical structures because of the focus on, and a priori hypotheses regarding small, difficult-toimage MRS structures such as the NAc and VTA/SN. Activation foci were superimposed on high-resolution T1-weighted images and their locations were interpreted using cortical (Duvernoy, 1995) and brainstem (Naidich et al., 2009) atlases. Anatomical localizations were cross-validated with the Mai atlas (Mai et al., 2008).

1.7.2.1. Effective connectivity analysis. The methods used here are similar to our previous study (Menon and Levitin, 2005). This analysis reflects a more elaborate examination of the interaction of multiple brain regions. Effective connectivity is defined as the influence of one region upon another, after discounting the influence of task-related effects as well as the effects of a common driving input (Friston et al., 1997). Effective connectivity is modeled here as a PPI (see Supplementary materials), in which the co-modulatory effects of the NAc-VTA/SN dopaminergic pathway are examined (Menon and Levitin, 2005). The individual time series for the NAC and VTA/SN were obtained by extracting the raw voxel time series in a sphere (6-mm radius) centered on the coordinates in the group activation maps (NAc: $12,10,-10$; VTA/SN: $-2,-22,-16$ ). Subjectspecific contrast images of music compared to scrambled music were determined and then entered into random effects group analyses using trait anhedonia as a covariate of interest. This secondlevel connectivity analysis allowed us to examine how effective connectivity of the MRS is correlated with individual differences in anhedonia. Additionally, we also examined the effective connectivity of the NAc alone, without the modulatory influences of the VTA. Significance of activation clusters were assessed using a voxelwise statistical height threshold of $p<.01$, with FWE corrections at the cluster level $p<.05$ ( $k=106$ voxels).

\section{Results}

\subsection{Behavioral}

Because participants were pre-screened to exclude history of or current depression and anxiety, the range of depression scores on the HAM-D was very small and the MASQ anxious arousal, general distress anxiety symptoms, and general distress depressive symptoms subscales as well as the MASQ-AD depression factor were not normally distributed (all Shapiro-Wilk $(21)>0.650, p<.003$ ). However, the MASQ-AD positive affect factor was normally distributed (Shapiro-Wilk $(21)=0.970, p=.723$ ).

\subsubsection{Convergent validity of MASQ-AD positive affect factor}

The MASQ-AD positive affect factor demonstrated convergent validity with the Chapman physical anhedonia scale (Chapman et al., 1976) in our sample $(r=0.530, p=.013)$.

\subsubsection{Relation between trait anhedonia and stimulus ratings}

Compared to Scrambled Music, participants rated clips in the Music condition as more pleasant $(t(17)=14.146, p<.001)$ and moving $(t(17)=-7.476, p<.001)$ (Table 2 ). Trait anhedonia was significantly negatively correlated with the pleasantness ratings of classical music (Fig. 1), suggesting that individuals with higher trait anhedonia levels found the music to be less pleasant overall. This relationship held true even after controlling for the participants' preference for classical music $(r=-0.573, p=.010)$, as measured by the Short Test of Music Preferences (Rentfrow and Gosling, 2003). Trait anhedonia was not correlated with pleasantness ratings in the Scrambled Music condition (Fig. 1). 
Trait anhedonia and music pleasantness

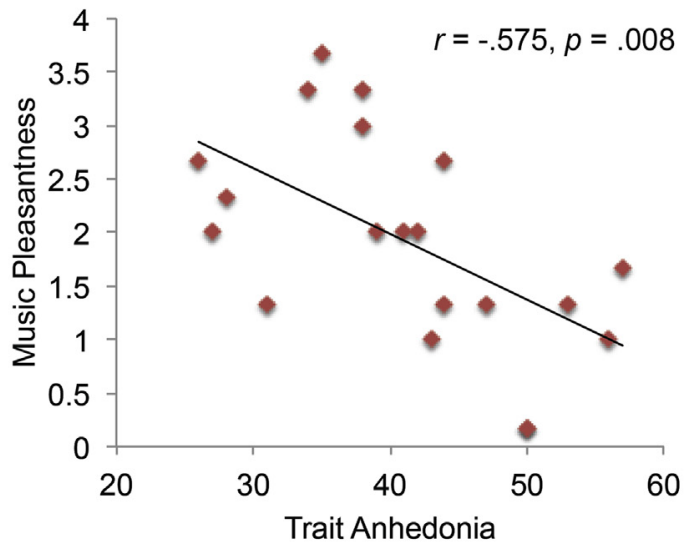

Trait anhedonia and scrambled music pleasantness

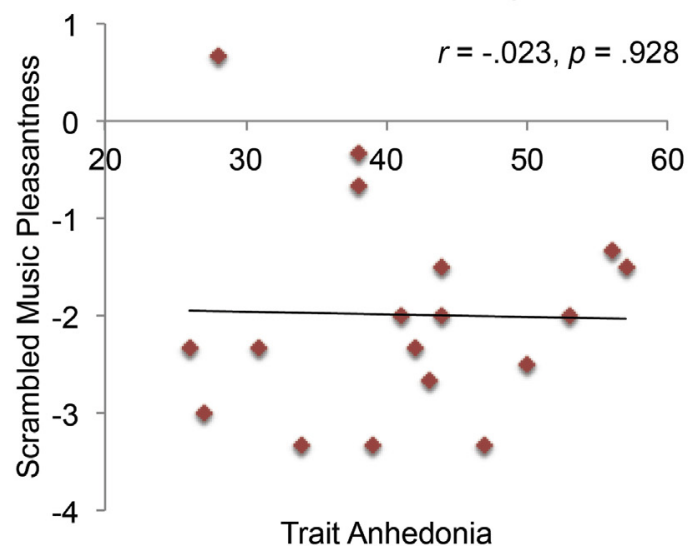

Fig. 1. Trait anhedonia was negatively correlated with music pleasantness ratings. Trait anhedonia, measured using the positive affect factor of the Mood and Anxiety Symptom Questionnaire anhedonic depression scale, was significantly negatively correlated with classical music pleasantness ratings but not with scrambled music pleasantness ratings.

\subsection{Brain imaging}

\subsubsection{Relation between brain activation and trait anhedonia}

We first confirmed that the MRS and related limbic and paralimbic areas associated with reward and affective information processing were activated during music listening. The music listening condition evoked significant activation in the MRS, including bilateral NAc and bilateral VTA/SN, as well as the left hypothalamus and bilateral amygdala. In addition, significant activation was found in paralimbic regions, including bilateral insula and OFC, as well as primary and secondary auditory cortices (Fig. 2, Table S1). Comparison with the Mai atlas (Mai et al., 2008) confirmed the involvement of the VTA/SN (Figure S1).

Next, we examined the relationship between trait anhedonia and brain response to Music contrasted with Scrambled Music. Trait anhedonia was negatively correlated with activation in right NAc, basal forebrain and bilateral hypothalamus (Fig. 3, Table 3). Significant negative correlations were also detected in the OFC, anterior insula, anterior and posterior cingulate cortex, and ventromedial prefrontal cortex (Fig. 4, Table 3). Additional analysis using a ROI from previously published study (Gottfried et al., 2003) confirm that the OFC $(r=-0.602, p=.004)$ is negatively correlated with trait anhedonia (Figure S2). Importantly, trait anhedonia was not correlated with auditory cortex responses. No brain regions showed positive correlations with trait anhedonia. Nearly identical results were obtained when using the entire MASQ-AD subscale.

\subsubsection{Relation between brain activation and music pleasantness ratings}

One subject was excluded for invalid music pleasantness ratings and one additional subject was excluded as an outlier. Amongst the remaining sample $(n=19)$, no brain regions showed significant positive or negative correlations with music pleasantness ratings.

\subsubsection{Relation between MRS effective connectivity and trait anhedonia}

To gain additional insights into the functional circuits mediating trait anhedonia, we examined effective connectivity of the MRS mediated by the NAc-VTA/SN dopaminergic pathway. To accomplish this, we first used PPI in each participant to determine NAcVTA/SN interactions during music, contrasted with spectrally matched scrambled music. The resulting contrast images were then subjected to a second-level regression analysis using trait anhedonia as a covariate. This analysis provides insights into music taskrelated influences of the MRS on all other brain regions by estimating connectivity after discounting the influence of overall taskrelated activation and the effects of common driving inputs. Trait anhedonia was negatively correlated with MRS effective connectivity in the right superior temporal gyrus, right auditory cortex, left OFC and bilateral insula (Fig. 5, Table 4) as well as paracingulate gyrus, frontal operculum, inferior and middle temporal gyrus, secondary somatosensory cortex and supramarginal gyrus. In a second analysis we examined effective connectivity of the NAc without incorporating its interaction with the VTA/SN. In this case, no brain regions were significantly correlated with effective connectivity of the NAc (Fig. 5, Table 4).

\section{Discussion}

In this study we examined how brain response and connectivity is impacted by trait anhedonia. A unique aspect of our study is that trait anhedonia was examined in a well-characterized and methodically assessed group of normal healthy adults with no history of psychiatric illness. Although anhedonia is most often associated with major psychiatric disorders, our findings suggest that dimensional approaches to trait anhedonia can provide important insights into its neurobiological underpinnings. Behaviorally, our findings indicate that perceived pleasantness of ecologically-relevant "real-world" auditory stimuli is reduced in trait anhedonia. In conjunction with this behavioral finding, we found that brain reactivity and effective connectivity of brain systems involved in reward processing are negatively impacted by trait anhedonia.

\subsection{Reactivity of MRS and related limbic structures is negatively impacted by trait anhedonia}

A novel finding of our study is that, even in a well-characterized group of healthy adults, trait anhedonia is associated with reduced reactivity in MRS and related limbic regions. As predicted, we found that higher levels of trait anhedonia were associated with reduced activity in the NAc. Although the VTA/SN was activated during music listening, we were not able to detect anhedonia-related effects in this dopaminergic midbrain region. Instead we found that higher levels of trait anhedonia were associated with reduced activity in other key MRS and limbic structures, including the basal forebrain, ventral striatum and hypothalamus, which are linked to the VTA by the MFB (Coenen et al., 2009, 2012, 2011). Electrophysiological studies in animals and human fMRI studies have suggested that the ventral striatum and basal forebrain encode motivational salience of sensory stimuli leading to increased attention to salient stimuli (Haber and Knutson, 2010; Lin and 


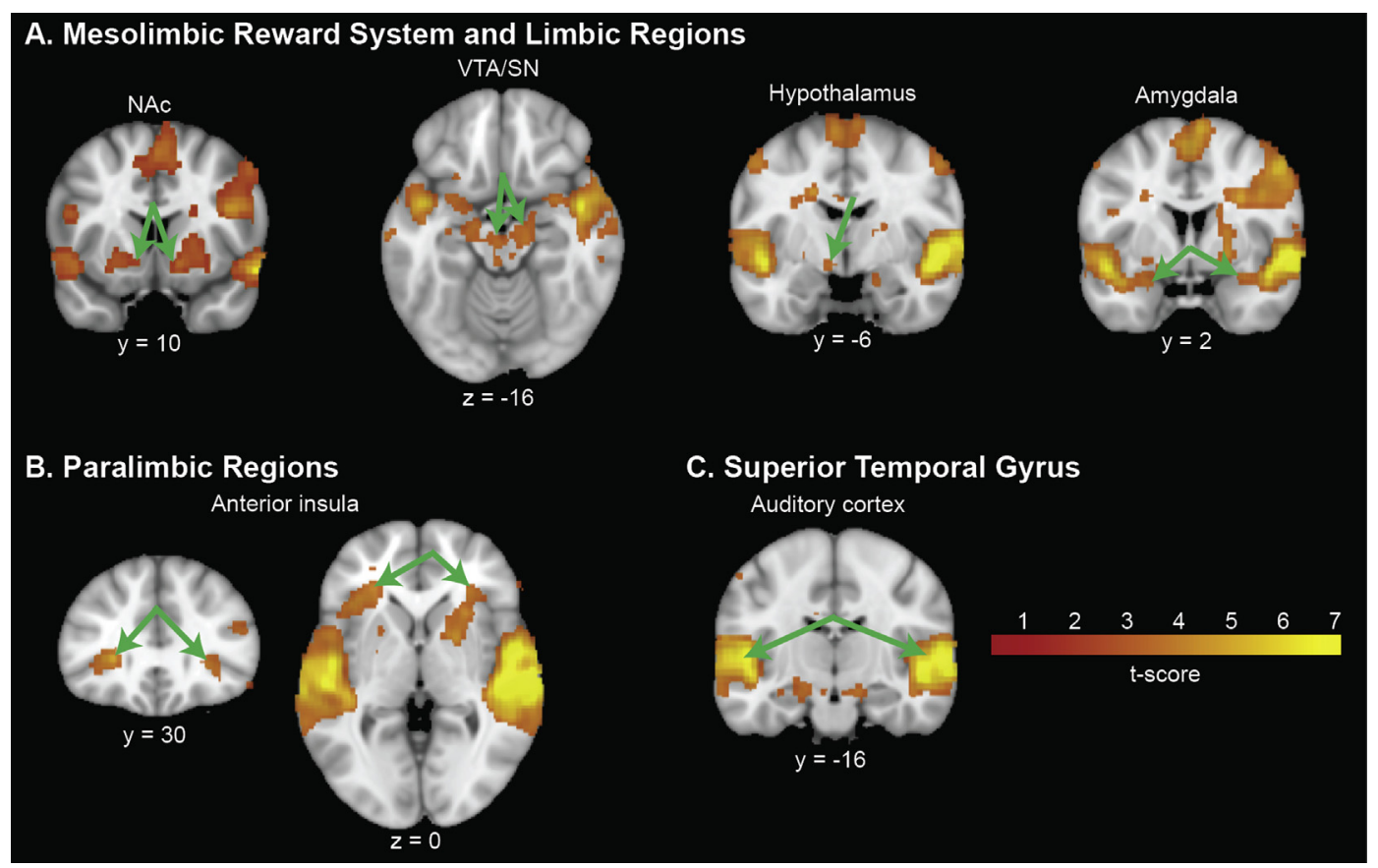

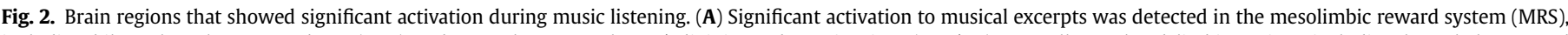

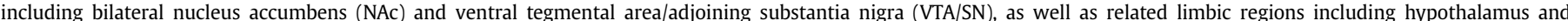

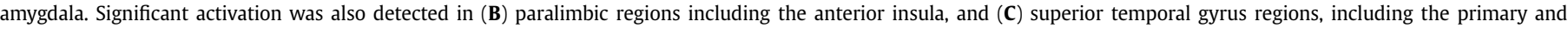
secondary auditory cortices. MNI coordinates of each slice are shown in mm.

Nicolelis, 2008; Pessiglione et al., 2007). The location of the basal forebrain cluster in our study was close to the area reported by Pessiglione et al. (2007) in their study of motivational salience involving monetary rewards. The basal forebrain, in particular, is one of the largest neuromodulatory structures in the mammalian brain with widespread projections to cortical areas including the OFC, and subcortical areas including the NAc and dopaminergic neurons in the VTA/SN (Semba, 2000). The NAc and ventral striatum, core structures of the MRS, are known to be activated by a wide range of pleasurable stimuli, including monetary rewards (Knutson et al., 2001), addictive drugs (Robinson and Berridge, 2000), food (Small et al., 2001), and music (Menon and Levitin, 2005). Our findings extend previous studies which found that music elicits strong MRS response (Blood and Zatorre, 2001; Koelsch et al., 2006; Menon and Levitin, 2005), and show that MRS reactivity is related to trait anhedonia even after controlling for

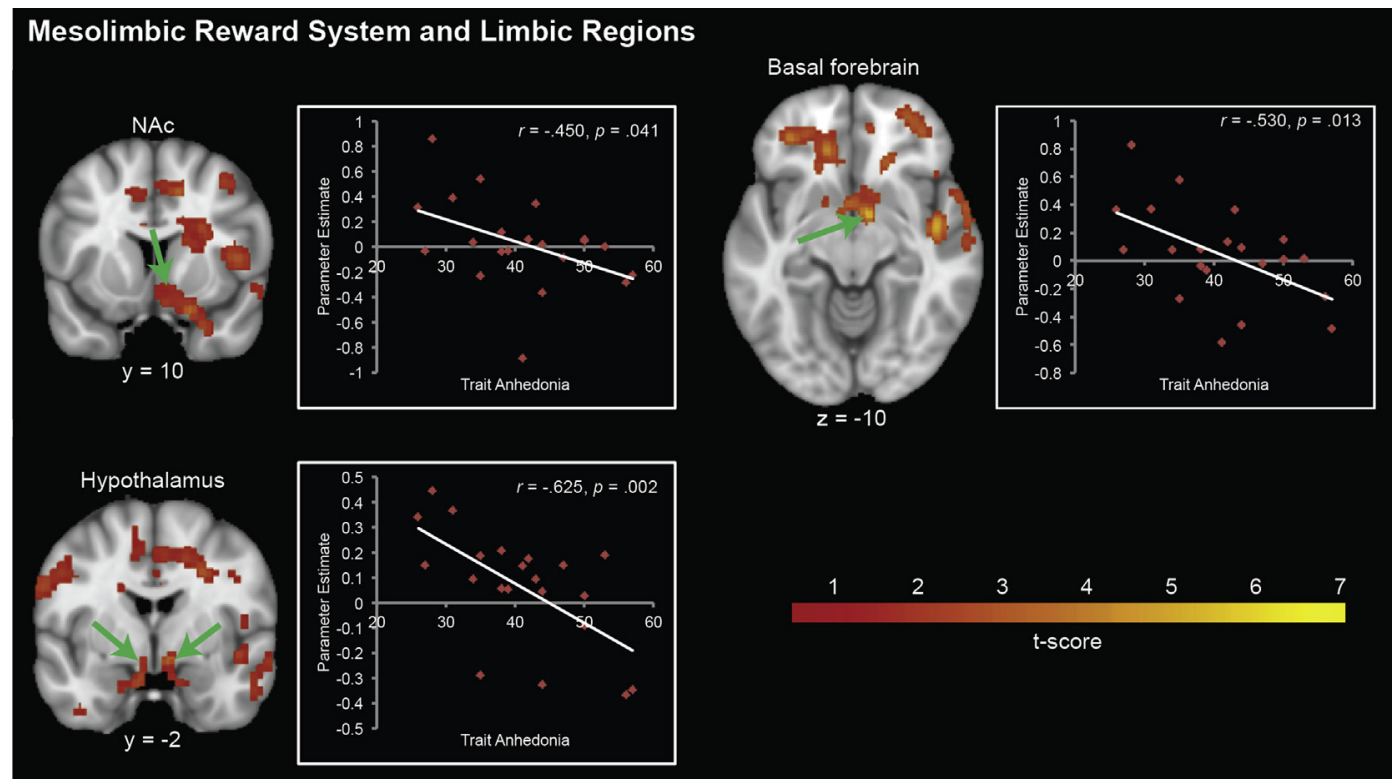

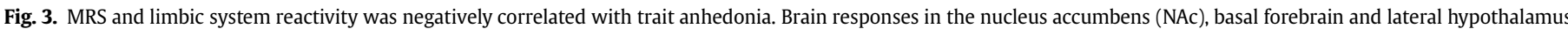

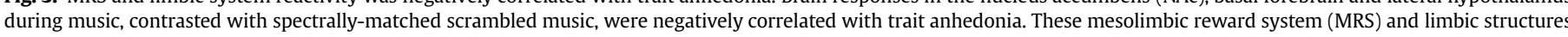

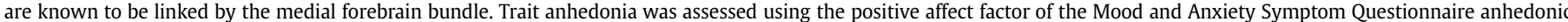

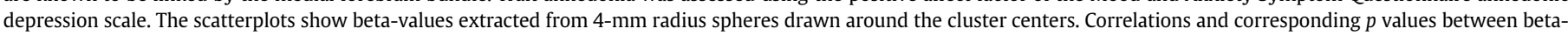
values and individual anhedonia scores are also shown. 
Table 3

Brain areas negatively correlated with trait anhedonia.

\begin{tabular}{|c|c|c|c|c|c|}
\hline & \multirow[t]{2}{*}{$\begin{array}{l}\text { Peak } \\
\text { T-score }\end{array}$} & \multirow[t]{2}{*}{$\begin{array}{l}\text { Size of cluster } \\
\text { (voxels) }\end{array}$} & \multicolumn{3}{|c|}{$\begin{array}{l}\text { Peak MNI } \\
\text { coordinates } \\
(\mathrm{mm})\end{array}$} \\
\hline & & & $X$ & Y & $Z$ \\
\hline \multicolumn{6}{|c|}{ Mesolimbic reward system and limbic regions } \\
\hline $\mathrm{R}$ basal forebrain & 4.15 & & 8 & 0 & -10 \\
\hline $\mathrm{R}$ hypothalamus & 3.11 & & 10 & -2 & -12 \\
\hline $\mathrm{R}$ nucleus accumbens & 2.08 & & 8 & 10 & -8 \\
\hline \multicolumn{6}{|l|}{ Paralimbic regions } \\
\hline $\begin{array}{l}\text { R ventromedial prefrontal } \\
\text { cortex }\end{array}$ & 4.72 & 120 & 10 & 40 & -16 \\
\hline $\mathrm{R}$ anterior cingulate cortex & 4.70 & 253 & -4 & 14 & 24 \\
\hline L orbital frontal cortex & 4.10 & 183 & -20 & 40 & -14 \\
\hline $\begin{array}{l}\mathrm{R} \text { paracingulate gyrus } \\
\mathrm{R} \text { insula }\end{array}$ & 3.67 & 122 & 14 & 10 & 44 \\
\hline \multicolumn{6}{|l|}{ Cortical regions } \\
\hline $\mathrm{R}$ middle frontal gyrus & 4.48 & 379 & 36 & 18 & 42 \\
\hline $\mathrm{R}$ angular gyrus & 4.55 & 273 & 44 & -58 & 40 \\
\hline L supramarginal gyrus & 4.02 & 130 & -52 & -32 & 52 \\
\hline $\begin{array}{l}\mathrm{R} \text { inferior frontal gyrus Pars } \\
\text { opercularis }\end{array}$ & 3.86 & 113 & 48 & 14 & 8 \\
\hline Frontal pole & 3.63 & 122 & 2 & 62 & 10 \\
\hline
\end{tabular}

auditory processing using spectrally well-matched scrambled musical excerpts. Although the primary and secondary auditory cortices showed high levels of activation during music listening, responses in these regions were not associated with trait anhedonia. Amongst the depression and anhedonia scales, only anhedonia demonstrated normal variability and a significant relationship to MRS activity. Furthermore, although trait anhedonia was significantly negatively correlated with music pleasantness ratings, no brain regions showed significant correlations with music pleasantness ratings during music listening, demonstrating a specific relationship between trait anhedonia and MRS activity. Thus, our neuroimaging findings provide direct empirical support for the role of anhedonia in the brain's response to pleasurable and rewarding stimuli in the absence of psychopathology.

\subsection{Reactivity of paralimbic structures is negatively impacted by trait anhedonia}

In addition to the MRS, responses in paralimbic areas were also negatively correlated with trait anhedonia. Paralimbic areas, most notably the insula, OFC and cingulate cortex are known to be involved in higher-order processing of both positively and negatively valenced affective stimuli. The anterior insula cortex is involved in subjective feelings associated with salient emotional stimuli (Craig, 2009). This region has also been hypothesized to anchor a "saliency network" which plays a crucial role in detecting and coordinating attentional response to salient stimuli (Seeley et al., 2007), suggesting that attention to salient pleasurable stimuli is adversely affected by trait anhedonia. The anterior insula also interacts with the midand posterior-sections of the insular cortex as well as the hypothalamus to map sensory stimuli to distinct physiological sensations (Craig, 2003). Although the mechanisms by which this system regulates physiological responses, such as frisson, shivers, and heart rate changes, that can accompany music listening (Blood and Zatorre, 2001; Panksepp, 1995) is currently not well understood, our findings nevertheless suggest that anterior insula dysfunction related to trait anhedonia may reduce physiological reactivity to music and similar positively valenced environmental stimuli.

OFC responses were also strongly negatively associated with trait anhedonia and confirmatory analyses using a priori ROIs based on previous studies confirmed that the OFC is strongly negatively

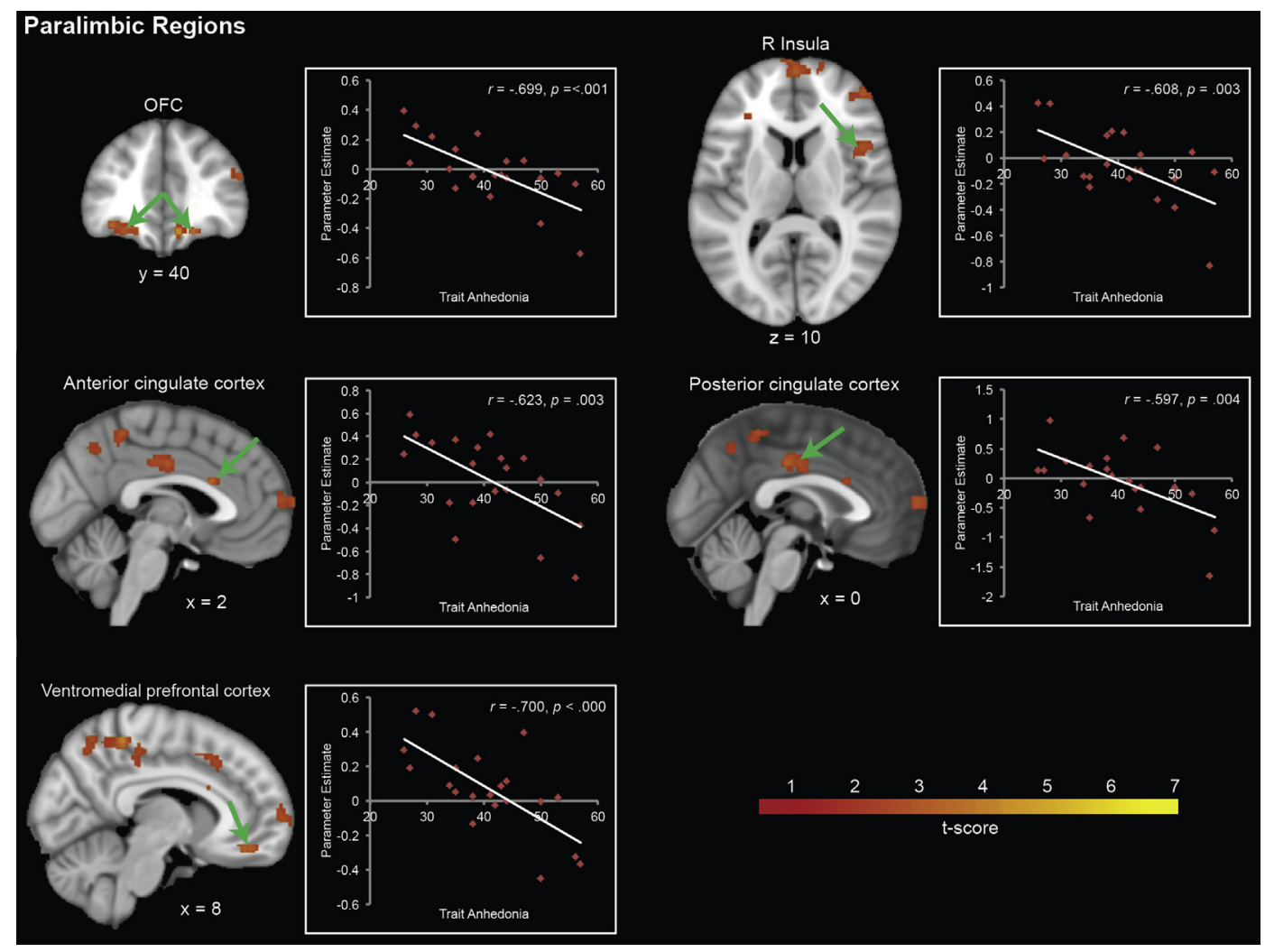

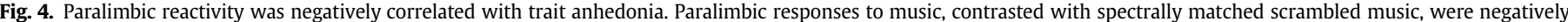

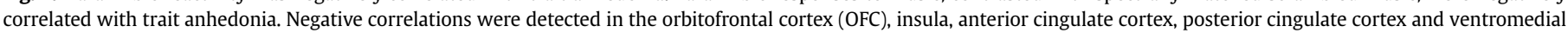
prefrontal cortex. Other details as in Fig. 3. 


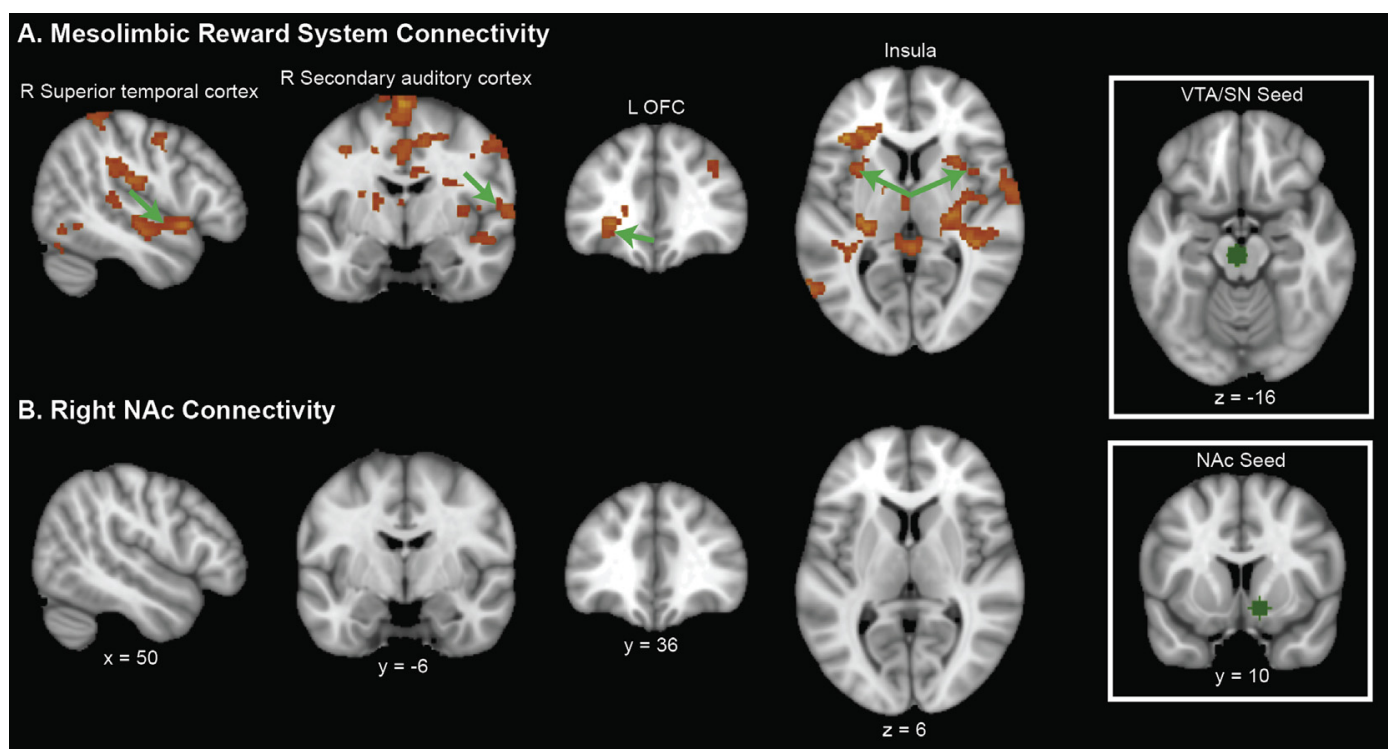

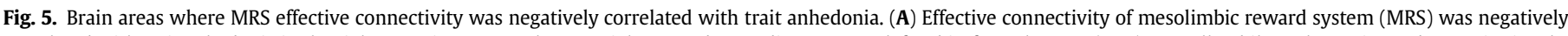

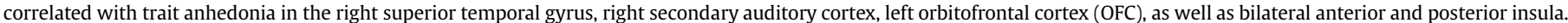

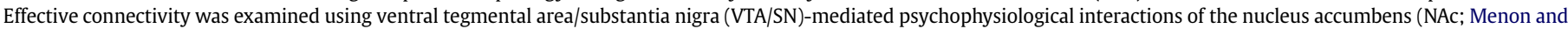

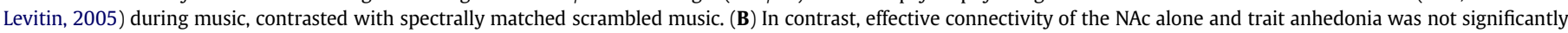
correlated in any brain region.

correlated with anhedonia (Chiavaras et al., 2001; Gottfried et al., 2003). These OFC areas are thought to be more important for cognitive evaluation and regulation of affective stimuli, rather than generating core reward-related signals to pleasant events (Berridge and Kringelbach, 2008; Dickinson and Balleine, 2008). In addition to reciprocal connections with the anterior insula (Kringelbach, 2005), the OFC is also connected to the MRS through the MFB and adjacent anterior thalamic radiation, and these fiber tracts are thought to be important for maintaining affective equilibrium (Coenen et al., 2012). Each of these regions was negatively modulated by trait anhedonia, further suggesting that the coordination of sensory, reward and hedonic experiences that accompany positively valenced stimuli such as music is impaired by trait anhedonia.

\subsection{Brain connectivity of core MRS structures with paralimbic areas is modulated by trait anhedonia}

Connectivity analysis provided novel insights into impaired functional circuits underlying trait anhedonia, and two analyses

\section{Table 4}

Brain areas that showed significant negative correlations between trait anhedonia and effective connectivity of the mesolimbic reward system (MRS). Effective connectivity of the MRS examined interactions of the right nucleus accumbens (NAc; 12 , $10,-10)$ and ventral tegmental area/substantia nigra (VTA/SN; $-2,-22,-16$ ) with other brain regions. No regions showed significant positive correlations between trait anhedonia and MRS effective connectivity.

\begin{tabular}{llllrr}
\hline & $\begin{array}{l}\text { Peak } \\
T \text {-Score }\end{array}$ & $\begin{array}{l}\text { Size of cluster } \\
\text { (voxels) }\end{array}$ & \multicolumn{3}{l}{$\begin{array}{l}\text { Peak MNI } \\
\text { coordinates }(\mathrm{mm})\end{array}$} \\
\cline { 4 - 6 } & & & $X$ & $Y$ & $Z$ \\
\hline L insula & 5.79 & 391 & -24 & 32 & 0 \\
L orbitofrontal cortex & 2.74 & & -28 & 36 & -10 \\
R inferior parietal lobule & 5.70 & 2465 & 62 & -28 & 32 \\
R secondary auditory cortex & 3.70 & & 64 & -4 & 6 \\
R superior temporal cortex & 4.38 & & 52 & 10 & -8 \\
R insula & 3.14 & & 40 & 8 & 6 \\
R lateral occipital cortex & 5.59 & 365 & 60 & -60 & -6 \\
L cerebellum & 5.56 & 366 & -48 & -58 & -38 \\
L premotor cortex & 5.26 & 2300 & -14 & 0 & 74 \\
L parietal operculum & 5.22 & 2427 & -38 & -18 & 24 \\
\hline
\end{tabular}

and findings are noteworthy here. First, we examined the role of putative mesolimbic dopaminergic pathways by examining the conjoint PPI of the NAc and the VTA/SN, similar to our previous study (Menon and Levitin, 2005). This analysis probes for brain regions that showed greater temporal interactions with the NAc only at the specific time points when the VTA/SN is also co-active. Thus, we sought to find brain regions whose signal changes were synchronized with significant changes in both the NAc and the VTA/ SN. This allows us to draw inferences about MRS connectivity involving the key nodes of the dopaminergic reward pathway. We found that trait anhedonia negatively impacted MRS connectivity with several paralimbic areas including the bilateral insula and OFC (Fig. 5, Table 4). The strong negative correlations between the MRS and paralimbic areas are consistent with previous findings (Menon and Levitin, 2005) which suggest a tight link between the "affective" and "cognitive" sub-systems involved in music listening. Other cortical areas most notably the belt and parabelt areas of the right auditory cortex as well as other subdivisions of the superior temporal gyrus also showed reduced MRS related modulation in relation to trait anhedonia (Fig. 5, Table 4). Critically, when we examined effective connectivity of the NAc without incorporating its interactions with the VTA, no brain regions showed significant connectivity. Taken together, these results suggest that conjoint interactions in the dopaminergic NAc-VTA/SN MRS pathway play an important role in trait anhedonia by influencing responses in paralimbic areas that regulate emotional reactivity to hedonic stimuli (Berridge and Kringelbach, 2008).

\subsection{Implications for the conceptualization of trait anhedonia}

As discussed above, our findings suggest a degree of anatomical specificity in how anhedonia modulates brain response. In particular, trait anhedonia was associated with specific MRS and related limbic and paralimbic structures including the NAc, basal forebrain, hypothalamus, anterior insula and OFC. Although the temporal lobes showed the strongest levels of brain activation during music listening, responses in these areas were not correlated with trait anhedonia. Our findings provide novel evidence for the neural 
correlates of anhedonia in the absence of psychiatric illness. By including only participants without any history of psychiatric illness, we were able to examine normal variation of anhedonia and eliminate the potential confounds arising from depression, anxiety and other disorders.

Only one previous study has examined trait anhedonia and MRS reactivity in a similar healthy sample. Harvey et al. (2007) found a significant positive correlation between anhedonia severity and activity in the ventromedial prefrontal cortex in the processing of positively valenced information. They did not, however, find a relationship between NAc activity and trait anhedonia. The discrepancy between our findings and those reported by Harvey and colleagues may be partly due to the use of the positive affect factor of the MASQ-AD, a more recently developed and theoretically driven measure of anhedonia (Watson et al., 1995a, 1995b), as well as the more emotive musical stimuli used in the present study. In comparison to viewing pleasant pictures, listening to music evokes strong emotions, including intense feelings of frisson and pleasure (Huron, 2006; Krumhansl, 1997; Salimpoor et al., 2011; Sloboda and Juslin, 2001). Our findings of NAc reactivity are more consistent with the study by Wacker and colleagues (2009) who found a similar pattern of negative reactivity in relation to anhedonia in the NAC using the MASQ-AD, albeit in a group that included individuals with psychiatric conditions. Our findings further converge with treatment studies that have shown that deep brain stimulation to the NAc alleviates anhedonia symptoms in treatment-resistant depression by increasing reward seeking behaviors (Schlaepfer et al., 2008).

\subsection{Limitations and future directions}

Although our study provides novel insights into brain structures and circuits affected by trait anhedonia, several limitations are important to note here. First, the spatial resolution was not adequate for determining the precise anatomical localization of brainstem and basal forebrain structures that were negatively correlated with trait anhedonia. However, the VTA/SN was clearly activated during music listening (Fig. 2, Table S1) and response in the basal forebrain was negatively correlated with anhedonia. Second, the relationship between brain activity and trait anhedonia was not examined using sad or melancholic music pieces. Some of the brain structures reported in our study, particularly paralimbic regions, have also been shown to be activated by negatively valenced stimuli (Mitterschiffthaler et al., 2007; Murray, 2007; Yang et al., 2002), and without sad music pieces we are unable to specify whether the activations reported here were specific to the experience of positively valenced rewarding stimuli.

Replication of this study using methods with higher spatial resolution as well as both positively and negatively valenced stimuli will be important for increasing our understanding of the neurobiological basis of anhedonia. Future work examining clinical populations will also be important for determining whether the same neural patterns seen in trait anhedonia are also seen in psychiatric disorders. Finally, studies distinguishing between reward seeking and pleasure are important to further refine our understanding of the brain's reward system and anhedonia. As an example, the MFB, which was recently identified in humans using diffusion tensor imaging (Coenen et al., 2009), has been linked to depression (Coenen et al., 2012, 2011; Schoene-Bake et al., 2010). The MFB is specifically related to reward seeking (Coenen et al., 2011), and this may be directly linked to recent conceptualizations of anhedonia subtypes such as motivational and consummatory anhedonia (Treadway et al., 2009; Treadway and Zald, 2010).

\subsection{Conclusions}

Although anhedonia is a prominent feature of many psychiatric disorders, our study demonstrates that the neural effects of trait anhedonia can also be observed dimensionally in healthy individuals. Our findings provide new insights into brain structures and functional MRS/MFB circuits underlying the neurobiological basis of anhedonia. Examining the dimensional aspects of anhedonia has the potential to better inform the treatment of anhedonia as a clinical symptom in disorders such as depression, schizophrenia, and bipolar disorder. Importantly, anhedonia symptoms in these illnesses are inadequately treated by available pharmacological or psychosocial interventions (Horan et al., 2006; Nutt et al., 2007), and residual anhedonia is associated with high levels of morbidity (Buckner et al., 2008). A recent study found that psychotherapy designed to increase engagement with rewarding stimuli improved activation of the MRS system in depression (Dichter et al., 2009). Better characterization of MRS reactivity and connectivity can therefore serve as a useful probe to assess treatments designed to ameliorate the negative impact of anhedonia. Finally, our study provides an important template against which various subtypes of anhedonia such as general, physical and social anhedonia, as well as consummatory, motivational, and decisional anhedonia (Treadway et al., 2009; Treadway and Zald, 2010) can be examined. Evaluating these subtypes in the context of healthy as well as psychiatric populations will allow us to further characterize the dimensional and categorical aspects of anhedonia.

\section{Contributors}

JK, DJL and VM designed the study and wrote the protocol. CBY, EK and KP performed the statistical analysis. JK, CBY and VM wrote the manuscript. All authors contributed to and have approved the final manuscript.

\section{Funding body}

This work was supported in part by grants from the National Alliance for Research on Schizophrenia and Depression (NARSAD) Young Investigator Award to JK; the National Science Foundation Graduate Research Fellowship (\#DGE-0824162) to CBY; and a grant from National Science Foundation (\#BCS0449927) to VM and DJL.

\section{Conflict of interest}

All authors declare that they have no conflict of interest.

\section{Acknowledgments}

We thank Sarah Wu for assistance with data acquisition as well as the reviewers for their valuable feedback and suggestions.

\section{Appendix A. Supplementary material}

Supplementary material related to this article can be found at http://dx.doi.org/10.1016/j.jpsychires.2013.05.015.

\section{References}

Barkham M, Rees A, Shapiro DA, Stiles WB, Agnew RM, Halstead J, et al. Outcomes of time-limited psychotherapy in applied settings: replicating the Second Sheffield Psychotherapy Project. Journal of Consulting and Clinical Psychology 1996;64(5):1079-85.

Berridge KC, Kringelbach ML. Affective neuroscience of pleasure: reward in humans and animals. Psychopharmacology 2008;199(3):457-80. 
Blood AJ, Zatorre RJ. Intensely pleasurable responses to music correlate with activity in brain regions implicated in reward and emotion. Proceedings of the National Academy of Science 2001;98(20):11818-23.

Blood AJ, Zatorre RJ, Bermudez P, Evans AC. Emotional responses to pleasant and unpleasant music correlate with activity in paralimbic brain regions. Nature Neuroscience 1999;2(4):382-7.

Buckholtz JW, Treadway MT, Cowan RL, Woodward ND, Li R, Ansari MS, et al. Dopaminergic network differences in human impulsivity. Science 2010;329(5991):532.

Buckner JD, Joiner Jr TE, Pettit JW, Lewinsohn PM, Schmidt NB. Implications of the DSM's emphasis on sadness and anhedonia in major depressive disorder. Psychiatry Research 2008;159(1-2):25-30.

Chapman LJ, Chapman JP, Raulin ML. Scales for physical and social anhedonia. Journal of Abnormal Psychology 1976;85(4):374-82.

Chiavaras MM, LeGoualher G, Evans A, Petrides M. Three-dimensional probabilistic atlas of the human orbitofrontal sulci in standardized stereotaxic space. Neurolmage 2001;13(3):479-96.

Coenen VA, Honey CR, Hurwitz T, Rahman AA, McMaster J, Burgel U, et al. Medial forebrain bundle stimulation as a pathophysiological mechanism for hypomania in subthalamic nucleus deep brain stimulation for Parkinson's disease. Neurosurgery 2009;64(6):1106-14.

Coenen VA, Panksepp J, Hurwitz TA, Urbach H, Madler B. Human medial forebrain bundle (MFB) and anterior thalamic radiation (ATR): imaging of two major subcortical pathways and the dynamic balance of opposite affects in understanding depression. The Journal of Neuropsychiatry and Clinical Neuroscience 2012;24(2):223-36.

Coenen VA, Schlaepfer TE, Maedler B, Panksepp J. Cross-species affective functions of the medial forebrain bundle-implications for the treatment of affective pain and depression in humans. Neuroscience and Biobehavioral Reviews 2011;35(9):1971-81.

Craig AD. Interoception: the sense of the physiological condition of the body. Current Opinions in Neurobiology 2003;13(4):500-5.

Craig AD. How do you feel-now? The anterior insula and human awareness. Nature Reviews Neuroscience 2009;10(1):59-70.

Der-Avakian A, Markou A. The neurobiology of anhedonia and other reward-related deficits. Trends in Neurosciences 2012;35(1):68-77.

Dichter GS, Felder JN, Petty C, Bizzell J, Ernst M, Smoski MJ. The effects of psychotherapy on neural responses to rewards in major depression. Biological Psychiatry 2009;66(9):886-97.

Dickinson A, Balleine B. Hedonics: the cognitive-motivational interface. In: Kringelbach ML, Berridge KC, editors. Pleasures of the brain. Oxford, UK: Oxford University Press; 2008.

Dunn RT, Kimbrell TA, Ketter TA, Frye MA, Willis MW, Luckenbaugh DA, et al. Principal components of the Beck Depression Inventory and regional cerebral metabolism in unipolar and bipolar depression. Biological Psychiatry 2002;51(5):387-99.

Duvernoy HM. The human brain sten and ceregellum: surface, structure, vascularization, and three-dimensional sectional anatomy with MRI. New York: Springer-Verlag; 1995.

First MB, Spitzer RL, Gibbon M, Williams JBW. Structured clinical interview for DSM-IV-TR Axis I disorders - patient edition (SCID-I/P, 2/2001 revision). Washington, D.C.: American Psychiatric Press, Inc; 1997.

Franken IH, Rassin E, Muris P. The assessment of anhedonia in clinical and nonclinical populations: further validation of the Snaith-Hamilton Pleasure Scale (SHAPS). Journal of Affective Disorders 2007:99(1-3):83-9.

Friston KJ, Buechel C, Fink GR, Morris J, Rolls E, Dolan RJ. Psychophysiological and modulatory interactions in neuroimaging. Neurolmage 1997;6(3):218-29.

Glover GH, Lai S. Self-navigated spiral fMRI: interleaved versus single-shot. Magnetic Resonance in Medicine 1998;39(3):361-8.

Gottfried JA, O'Doherty J, Dolan RJ. Encoding predictive reward value in human amygdala and orbitofrontal cortex. Science 2003;301(5636):1104-7.

Haber SN, Knutson B. The reward circuit: linking primate anatomy and human imaging. Neuropsychopharmacology 2010;35(1):4-26.

Hamilton M. A rating scale for depression. Journal of Neurology, Neurosurgery, and Psychiatry 1960;23:49-65.

Harvey PO, Pruessner J, Czechowska Y, Lepage M. Individual differences in trait anhedonia: a structural and functional magnetic resonance imaging study in non-clinical subjects. Molecular Psychiatry 2007;12(8): 703, 767-775.

Horan WP, Kring AM, Blanchard JJ. Anhedonia in schizophrenia: a review of assessment strategies. Schizophrenia Bulletin 2006;32(2):259-73.

Huron D. Sweet anticipation: music and the psychology of expectation. Cambridge, MA: MIT Press: 2006.

Juslin P, Sloboda JA. Music and emotion: theory and research. Oxford: Oxford University Press; 2001

Keedwell PA, Andrew C, Williams SC, Brammer MJ, Phillips ML. The neural correlates of anhedonia in major depressive disorder. Biological Psychiatry 2005;58(11):843-53.

Knutson B, Fong GW, Adams CM, Varner JL, Hommer D. Dissociation of reward anticipation and outcome with event-related fMRI. NeuroReport 2001;12(17): 3683-7.

Koelsch S, Fritz T, V. C. DY, Muller K, Friederici AD. Investigating emotion with music: an fMRI study. Human Brain Mapping 2006;27(3):239-50.

Kopta SM, Howard KI, Lowry JL, Beutler LE. Patterns of symptomatic recovery in psychotherapy. Journal of Consulting and Clinical Psychology 1994;62(5): 1009-16.
Kringelbach ML. The human orbitofrontal cortex: linking reward to hedonic experience. Nature Reviews Neuroscience 2005;6(9):691-702.

Krumhansl CL. An exploratory study of musical emotions and psychophysiology. Canadian Journal of Experimental Psychology 1997;51(4):336-53.

Lane RD, Reiman EM, Bradley MM, Lang PJ, Ahern GL, Davidson RJ, et al. Neuroanatomical correlates of pleasant and unpleasant emotion. Neuropsychologia 1997;35(11):1437-44.

Lin SC, Nicolelis MA. Neuronal ensemble bursting in the basal forebrain encodes salience irrespective of valence. Neuron 2008;59(1):138-49.

Mai JK, Paxinos G, Voss T. Atlas of the human brain. 3rd ed. New York: Elsevier Inc; 2008

Menon V, Levitin DJ. The rewards of music listening: response and physiologica connectivity of the mesolimbic system. Neuroimage 2005;28(1):175-84.

Mesulam MM. From sensation to cognition. Brain 1998;121:1013-52.

Mitterschiffthaler MT, Fu CH, Dalton JA, Andrew CM, Williams SC. A functional MR study of happy and sad affective states induced by classical music. Human Brain Mapping 2007;28(11):1150-62.

Mobbs D, Greicius MD, Abdel-Azim E, Menon V, Reiss AL. Humor modulates the mesolimbic reward centers. Neuron 2003;40(5):1041-8.

Murray EA. The amygdala, reward and emotion. Trends in Cognitive Science 2007:11(11):489-97.

Naidich TP, Duvernoy HM, Delman BN, Sorensen AG, Kollias SS, Haacke EM. Duvernoy's atlas of the human brain stem and cerebellum. Springer; 2009.

Nitschke J, Heller W, Imig J, McDonald R, Miller G. Distinguishing dimensions of anxiety and depression. Cognitive Therapy and Research 2001;25(1):1-22.

Nutt D, Demyttenaere K, Janka Z, Aarre T, Bourin M, Canonico PL, et al. The other face of depression, reduced positive affect: the role of catecholamines in causation and cure. Journal of Psychopharmacology 2007;21(5):461-71.

Padoa-Schioppa C, Cai X. The orbitofrontal cortex and the computation of subjective value: consolidated concepts and new perspectives. Annals of the New York Academy of Sciences 2011;1239:130-7.

Panksepp J. The emotional sources of "chills" induced by music. Music Perception 1995;13(2):171-207.

Pessiglione M, Schmidt L, Draganski B, Kalisch R, Lau H, Dolan R, et al. How the brain translates money into force: a neuroimaging study of subliminal motivation. Science 2007;316(5826):904-6.

Rentfrow PJ, Gosling SD. The do re mi's of everyday life: the structure and personality correlates of music preferences. Journal of Personality and Social Psychology 2003;84(6):1236-56.

Robinson TE, Berridge KC. The psychology and neurobiology of addiction: an incentive-sensitization view. Addiction 2000;95:S91-117.

Salimpoor VN, Benovoy M, Larcher K, Dagher A, Zatorre RJ. Anatomically distinct dopamine release during anticipation and experience of peak emotion to music Nature Neuroscience 2011;14(2):257-62.

Schlaepfer TE, Cohen MX, Frick C, Kosel M, Brodesser D, Axmacher N, et al. Deep brain stimulation to reward circuitry alleviates anhedonia in refractory major depression. Neuropsychopharmacology 2008;33(2):368-77.

Schoene-Bake JC, Parpaley Y, Weber B, Panksepp J, Hurwitz TA, Coenen VA. Tractographic analysis of historical lesion surgery for depression. Neuropsychopharmacology 2010;35(13):2553-63.

Seeley WW, Menon V, Schatzberg AF, Keller J, Glover GH, Kenna H, et al. Dissociable intrinsic connectivity networks for salience processing and executive control. Journal of Neurosciences 2007;27(9):2349-56.

Semba K. Multiple output pathways of the basal forebrain: organization, chemica heterogeneity, and roles in vigilance. Behavioural Brain Research 2000;115(2): 117-41.

Shelton RC, Tomarken AJ. Can recovery from depression be achieved? Psychiatric Services 2001;52(11):1469-78.

Sloboda JA, Juslin P. Psychological perspectives on music and emotion. In: Juslin P, Sloboda JA, editors. Music and emotion: theory and research. Oxford: Oxford University Press; 2001. p. 71-104.

Small DM, Zatorre RJ, Dagher A, Evans AC, Jones-Gotman M. Changes in brain activity related to eating chocolate: from pleasure to aversion. Brain 2001;124 1720-33.

Treadway MT, Buckholtz JW, Schwartzman AN, Lambert WE, Zald DH. Worth the 'EEfRT'? the effort expenditure for rewards task as an objective measure of motivation and anhedonia. PLoS One 2009:4(8):e6598.

Treadway MT, Zald DH. Reconsidering anhedonia in depression: lessons from translational neuroscience. Neuroscience and Biobehavioral Reviews 2010;35(3):537-55.

Wacker J, Dillon DG, Pizzagalli DA. The role of the nucleus accumbens and rostra anterior cingulate cortex in anhedonia: integration of resting EEG, fMRI, and volumetric techniques. Neuroimage 2009;46(1):327-37.

Watson D, Clark LA, Weber K, Assenheimer JS, Strauss ME, McCormick RA. Testing a tripartite model: II. Exploring the symptom structure of anxiety and depression in student, adult, and patient samples. Journal of Abnormal Psychology 1995a;104(1):15-25.

Watson D, Weber K, Assenheimer JS, Clark LA, Strauss ME, McCormick RA. Testing a tripartite model: I. Evaluating the convergent and discriminant validity of anxiety and depression symptom scales. Journal of Abnormal Psychology 1995b;104(1):3-14.

Wolf DH. Anhedonia in schizophrenia. Current Psychiatry Reports 2006;8(4) 322-8.

Yang TT, Menon V, Eliez S, Blasey C, White CD, Reid AJ, Reiss AL. Amygdalar activation associated with positive and negative facial expressions. NeuroReport 2002; 13(14):1737-41. 